\title{
Validation of Discharge Diagnosis of Sickle Cell Disease Vaso-Occlusive Episodes in the French Hospital Electronic Database
}

\author{
Ondine Walter $\mathbb{D}^{1,2}$ \\ Julien Maquet $\mathbb{D}^{1,2}$ \\ Helene Derumeaux (D) $^{3}$ \\ Guillaume Moulis $\mathbb{D}^{1,2}$ \\ Margaux Lafaurie $\mathbb{1 D}^{2,4}$ \\ 'Service de Médecine Interne, Centre \\ Hospitalier Universitaire de Toulouse, \\ Toulouse, France; ${ }^{2}$ Centre d'Investigation \\ Clinique 1436, Centre Hospitalier \\ Universitaire de Toulouse, Toulouse, \\ France; ${ }^{3}$ Département d'Information \\ Médicale, Centre Hospitalier \\ Universitaire de Toulouse, Toulouse, \\ France; ${ }^{4}$ Service de Pharmacologie \\ Médicale et Clinique, Faculté de \\ Médecine, Centre Hospitalier \\ Universitaire de Toulouse, Toulouse, \\ France
}

Correspondence: Ondine Walter Service de Médecine Interne, pavillon URM, CHU Purpan, place du Dr Baylac,

TSA 4003 I, 31059, Toulouse Cedex 9, France

Tel +33561 779677

$\mathrm{Fax}+33561778058$

Email walter.o@chu-toulouse.fr
Keywords: sickle cell anemia, vaso occlusive crisis, predictive value of test, epidemiology

\section{Introduction}

Sickle cell disease (SCD) is the most frequent monogenic disease in the world (300,000 newborns per year). ${ }^{1}$ The prevalence depends on the geographical area, with predominance in sub-Saharan Africa, Mediterranean basin, and India. ${ }^{1}$ In France, about 500 newborns per year are diagnosed. ${ }^{2}$ SCD occurs in people who carry two abnormal alleles of the HBB gene, coding for the Beta chain of hemoglobin, located on chromosome 11. Depending on inherited mutations, there are several types of SCD. The main ones are: homozygous SS-SCD (71\% in France), double-heterozygous SC-SCD (22\%), and double-heterozygous S $\beta$-SCD (7\%). ${ }^{2}$

Patients with SCD present chronic hemolysis, recurrent episodes of pain (called acute vaso-occlusive crisis or acute vaso-occlusive episodes - VOEs), multiple organ dysfunction, and early death. ${ }^{1}$ VOE usually refers to different clinical subtypes such as painful vaso-occlusion, acute chest syndrome or acute splenic sequestration. VOEs are the leading cause of care-seeking in this population, affecting both patients with homozygous SS-SCD and those with double heterozygous SCD. They induce a decrease in quality of life and increased mortality. ${ }^{1}$

Population-based studies using health care or health insurance databases are very useful to assess the epidemiology and management of rare diseases like SCD in France. ${ }^{3}$

However, health insurance databases are primarily built for reimbursement purposes. Consequently, the identification of diseases encoded in these databases needs validation before use for research. The French Health Insurance System Database called SNDS (Système National des Données de Santé) virtually covers the entire French population ( $>66$ million inhabitants). It links administrative, outpatient health care, and in-patient data. In-patient data are collected in the PMSI (Programme de Médicalisation des Systèmes d'Informations) hospital database. It records data related to all hospital stays in both private and public hospitals, including admission and discharge dates, one primary discharge diagnosis (usually corresponding to the reason for hospitalization $) \pm$ one related discharge diagnosis, and associated discharge diagnoses (corresponding to comorbidities or events that occurred during the stay). These diagnoses are encoded at discharge using the 
International Classification of Diseases, 10th version (ICD-10th) by the physician in charge of the patient or by professional coding technicians reviewing the medical records. $^{3}$

In 2020, we conducted a cross-sectional study in the 2017 French PMSI database to assess the positive predictive value (PPV) of ICD-10 discharge diagnoses of hemolytic anemia. ${ }^{4}$ In this study, the PPV of sickle-cell disorders discharge diagnosis (including sickle-cell trait) was 97.6\% (confidence interval - CI: 95.0-100.0\%). However, the PPV of discharge diagnosis of VOEs was not assessed. This was the aim of this study.

\section{Materials and Methods}

We selected all hospital stays at Toulouse University Hospital, South of France (2860 beds) between January 2017 and December 2017 with a discharge diagnosis of VOEs, ie, with the D57.0 ICD-10 code. This code corresponds to "sickle-cell anemia with crisis". Albeit it is recommended to use this code for SSSCD with crisis, there is no other code for VOE in the ICD-10. In practice, all VOEs, including VOEs occurring in patients with double-heterozygous SCD, are encoded using this code.

True positive cases of VOE were ascertained by a detailed medical chart review. PPVs were calculated as the number of true positive cases divided by the number of patients with D57.0 discharge diagnosis code (ie, true-positive plus false-positive cases) and stratified according to the category of diagnosis code (primary, related and associated). Their 95\% Cis were calculated by the exact method (Clopper-Pearson interval). The demographics of our sample and the total population were compared by a Chi-squared test for categorical variables and a Student's $t$-test for quantitative variables. Analyses were conducted using SAS V9.4 ${ }^{\mathrm{TM}}$ (SAS Institute, Cary, NC, USA).

\section{Results}

During the study period, 882 discharge diagnosis of VOEs were identified, corresponding to 182 patients. The mean age of these patients at hospitalization was 23.1 years, $55.5 \%$ were women, $74.3 \%$ had SS-SCD genotype, $13.7 \%$ SC-SCD, and 8.2\% S $\beta$-SCD. We randomly selected 100 hospital stays for analysis, corresponding to 70 patients. Our sample was not different from the total population. The mean age was 22.0 years $(p=0.26)$, $62.9 \%$ were female $(\mathrm{p}=0.13)$, and the pattern of genotypes was SS-SCD 84.3\%, SC-SCD 10.0\%, and S $\beta$-SCD 4,2\% $(p=0.29)$. Among these 100 stays, 72 were true positive cases of VOE (including 10 patients with acute chest syndrome and 1 patient with acute splenic sequestration). Sixty-three concerned SS-SCD patients, 7 SC-SCD patients, and $2 \mathrm{~S} \beta-\mathrm{SCD}$ patients. False-positive cases $(n=28)$ were: scheduled transfusion $(n=14)$, annual organ dysfunction check-up $(n=3)$, scheduled surgical procedure $(n=1)$ and miscellaneous $(n=10)$. Among the 70 hospital stays with a VOE discharge diagnosis in the principal position, only one false-positive case was observed (abdominal pain in a patient with an SCD trait).

Overall, the PPV for VOE discharge diagnosis in the PMSI was $72.0 \%$, 95\% CI: 62.13-80.52\%. It was $98.57 \%$, 95\% CI: $92.29-99.96 \%$ for VOE as primary discharge diagnosis, $0 \%$ for related discharge diagnosis, and $33.3 \%$ for associated discharge diagnosis (Table 1).

\section{Discussion}

To the best of our knowledge, this study is the first to evaluate the PPVs of VOE discharge diagnosis in the French health database.

It was very high (98.6\%) for principal discharge diagnosis, while no true positive case of VOE was observed as related discharge diagnosis and few as associated discharge diagnosis. Consequently, epidemiological studies assessing VOE as an outcome should identify VOEs using primary discharge diagnosis only. Of note, this

Table I Positive Predictive Values of Vaso-Occlusive Episode Discharge Diagnosis in the French Hospital Database (D57.0 ICD-I0 Code)

\begin{tabular}{|l|l|l|l|}
\hline Category of Discharge Diagnosis & N & Number of True Positive Cases & PPV (95\% Cl) \\
\hline Overall & 100 & 72 & $72.0 \%(62.1-80.5)$ \\
Principal & 70 & 69 & $98.6 \%(92.3-100.0)$ \\
Related & 21 & 0 & - \\
Associated & 9 & 3 & $33.3 \%(7.5-70.1)$ \\
\hline
\end{tabular}

Abbreviations: $\mathrm{Cl}$, confidence interval; ICD-10, international classification of disease version I0; PPV, positive predictive value. 
study confirms that the D57.0 code was used to code VOEs whatever the type of SCD (SS, SC, or S $\beta$ ). Conversely, the consequence for epidemiology studies conducted in the SNDS is that it is impossible to distinguish VOEs by subtypes of SCD in this database.

Of note, Aljuburi et al conducted a study to assess trends in hospital admissions for patients with SCD, with or without VOE in the United Kingdom. ${ }^{5}$ They also used the D57.0 code as primary discharge diagnosis to identify VOE. However, the PPV had not been previously validated in this database. In 2016, Hansen et al assessed the PPV of hemolytic anemias diagnoses in the Danish National Patient Register. Among 25 patients with a D57 discharge diagnosis of SCD, 18 were true positive cases after medical chart review. However, they did not assess PPV of the D57.0 diagnosis due to the low number of patients with SCD in this study. ${ }^{6}$ In 2020 , Grosse et al reviewed all SCD identification algorithms used in USA insurance databases, with heterogeneous results. ${ }^{7}$ However, all these algorithms used ICD-9 diagnosis classification, preventing from any comparison with our findings.

The principal limitation of this study is that it was conducted in a single university center and may not reflect the performance of diagnosis coding at the national level. However, coding is similar in all private and public hospitals due to the application of validated national standards, consequently, it is expected that our results can be extrapolated to the whole SNDS. Secondly, acute chest syndrome or acute splenic sequestration cannot be distinguished among VOEs because there is no specific code in the ICD-10.

\section{Conclusion}

In conclusion, this study suggested the very high PPV of primary discharge diagnosis of VOE in the PMSI.

\section{Ethics Approval and Informed Consent}

This study was conducted in accordance with the Declaration of Helsinki. Studies assessing the accuracy of diagnosis coding by medical chart review are authorized by Toulouse University Hospital ethical committee. All hospitalized patients in this hospital received written information on the possibility of such studies.

\section{Author Contributions}

All authors contributed to data analysis, drafting or revising the article, gave final approval of the version to be published, and agree to be accountable for all aspects of the work.

\section{Funding}

This is an academic study, supported by grants from the University hospital of Toulouse and from the Société Nationale Française de Médecine Interne (SNFMI) and the Recherche et Enseignement en Médecine Interne (REMI) association.

\section{Disclosure}

Dr Guillaume Moulis received research grants and for participation to board from Amgen, Grifols, Novartis, Sobi, outside the submitted work. The authors report no other conflicts of interest in this work.

\section{References}

1. Piel FB, Steinberg MH, Rees DC. Sickle cell disease. $N$ Engl J Med. 2017;376(16):1561-1573. doi:10.1056/NEJMra1510865

2. Centre National de Coordination du Dépistage Néonatal. Rapport d'activité 2018 du Programme de dépistage néonatal; 2018. Available from: http://depistage-neonatal.org/wp-content/uploads/ 2019/12/Rapport-Activite-2018.pdf. Accessed April 19, 2021.

3. Moulis G, Lapeyre-Mestre M, Palmaro A, Pugnet G, Montastruc J-L, Sailler L. French health insurance databases: what interest for medical research? Rev Med Interne. 2015;36(6):411-417. doi:10.1016/j. revmed.2014.11.009

4. Maquet J, Derumeaux H, Lapeyre-Mestre M, Sailler L, Moulis G. Validation of hemolytic anemia discharge diagnosis codes in the French hospital database. Eur J Intern Med. 2020;79:136-138. doi:10.1016/j.ejim.2020.04.030

5. Aljuburi G, Laverty AA, Green SA, et al. Trends in hospital admissions for sickle cell disease in England, 2001/02-2009/10. J Public Health. 2012;34(4):570-576. doi:10.1093/pubmed/fds035

6. Hansen DL, Overgaard UM, Pedersen L, Frederiksen H. Positive predictive value of diagnosis coding for hemolytic anemias in the Danish national patient register. CLEP. 2016;8:241-252. doi:10.2147/ CLEP.S93643

7. Grosse SD, Green NS, Reeves SL. Administrative data identify sickle cell disease: a critical review of approaches in U.S. health services research. Pediatr Blood Cancer. 2020;67(12):e28703. doi:10.1002/ pbc. 28703 


\section{Publish your work in this journal}

Clinical Epidemiology is an international, peer-reviewed, open access, online journal focusing on disease and drug epidemiology, identification of risk factors and screening procedures to develop optimal preventative initiatives and programs. Specific topics include: diagnosis, prognosis, treatment, screening, prevention, risk factor modification,

Submit your manuscript here: https://www.dovepress.com/clinical-epidemiology-journal systematic reviews, risk \& safety of medical interventions, epidemiology \& biostatistical methods, and evaluation of guidelines, translational medicine, health policies \& economic evaluations. The manuscript management system is completely online and includes a very quick and fair peer-review system, which is all easy to use. 